\title{
La eficacia de la estimulación para el Alzheimer basada en Mindfulness (MBAS) en la progresión del deterioro cognitivo: un ensayo clínico aleatorizado a doble ciego
}

\author{
Domingo Jesús Quintana Hernández ${ }^{1}$ y María del Pino Quintana Montesdeoca ${ }^{2}$ \\ ${ }^{1}$ Asociación Canaria para el Desarrollo de la Salud a través de la Atención (España); \\ ${ }^{2}$ Universidad de Las Palmas de Gran Canaria (España)
}

Hemos llevado a cabo un ensayo clínico controlado aleatorizado, con el fin de investigar la viabilidad, seguridad y los efectos de la práctica de mindfulness en la evolución cognitiva de la enfermedad de Alzheimer (EA). La eficacia de MBAS con enfermos de Alzheimer se probó con un diseño de medidas repetidas con las escalas cognitivas del CAMDEX-R (CAMCOG y MMSE) cada seis meses durante dos años. En el estudio participaron 168 pacientes con Enfermedad de Alzheimer probable según los criterios NINCDS-ADRDA tratados con donepecilo. Se aleatorizaron los sujetos en tres grupos experimentales (estimulación cognitiva, relajación muscular progresiva y MBAS) y un grupo control. Cada grupo experimental trabajó en sesiones de 90 minutos, tres veces por semana durante dos años (96 semanas). Los resultados indicaron que MBAS mantuvo estable la función cognitiva a lo largo de dos años. Sin embargo el resto de grupos presentaron un deterioro significativo en la capacidad cognitiva. Por lo tanto, parece que la práctica de mindfulness, tal como se aplica en este programa, puede tener un papel preventivo en la progresión del deterioro cognitivo en la EA. Estos resultados apoyan el uso de mindfulness como tratamiento no farmacológico.

Palabras clave: Enfermedad de Alzheimer, envejecimiento, mindfulness, cognición, relajación, ECA.

The efficacy of Mindfulness-based Alzheimer's stimulation (MBAS) in the progression of cognitive impairment: a double-blind, randomized clinical trial. We conducted a randomized, controlled study in order to investigate the feasibility, security as well as the effects of the practice of mindfulness upon the evolution of AD. The efficacy of a Mindfulness-based Cognitive Stimulation program for Alzheimer's patients was tested in this double-blind, randomized clinical trial, using repeated measures of CAMDEX (CAMCOG y MMSE) every six months during two years. A total of 168 patients, presenting a Probable Alzheimer Disease according to NINCDS-ADRDA criteria and treated with donepezil, were randomly assigned to the four following non-pharmacological treatments: standard cognitive stimulation, progressive muscular relaxation, mindfulness-based Alzheimer stimulation and control group, which received no intervention. Each experimental group worked in 90-minute sessions, three times per week during two years (a total of 96 weeks). Results indicated that the Mindfulness-based Alzheimer Stimulation group did not show as much a significant decline of cognitive function as the other groups did. Therefore, it seems that mindfulness practice, as implemented in this program, can have a preventive role against the progression of cognitive impairment in $\mathrm{AD}$. These results support the use of mindfulness as a non-pharmacological treatment.

Keywords: Alzheimer Disease, Ageing, Mindfulness, Cognitive Stimulation, Progressive Muscular Relaxation, RCT.

Correspondencia: Domingo Jesús Quintana Hernández. Asociación Canaria para el Desarrollo de la Salud a través de la Atención. Las Palmas de Gran Canaria (España). E-mail: domingoj.quintana@gmail.com 
La enfermedad de Alzheimer (EA) no tiene cura actualmente. El tratamiento existente modifica temporalmente la sintomatología, aunque no detiene el curso neurodegenerativo de la enfermedad. En este contexto, mindfulness se presenta como una nueva esperanza para las personas mayores, familiares y cuidadores de quienes padecen esta enfermedad por las implicaciones que tiene su práctica como mecanismo neuroprotector y activador de la neuroplasticidad (Marciniak et al., 2014). En los últimos años se han llevado a cabo algunos estudios, entre los que destaca el de Wells et al. (2013) utilizando el programa de reducción de estrés basado en mindfulness (MBSR) de Kabat-Zinn (1990), que apuntan hacia esta posibilidad. Sin embargo, se están produciendo los primeros pasos para mostrar los efectos de mindfulness sobre la EA y con esta investigación se presenta el primer estudio longitudinal que utiliza mindfulness como tratamiento para retrasar el deterioro cognitivo.

La práctica de mindfulness se puede definir como la conciencia sin prejuicios de la experiencia en el momento presente con una actitud de aceptación y afecto (Bishop et al., 2004). Esta capacidad cognitiva requiere el desarrollo de una focalización atencional intencionada, que en nuestro estudio se ha entrenado mediante la participación conjunta del enfermo con un cuidador principal.

Los tratamientos basados en mindfulness han mostrado que durante su práctica se incrementa la irrigación cerebral en áreas corticales y subcorticales, incluido el hipocampo (Lazar et al., 2005; Hölzel et al., 2011), aumenta la conectividad y la densidad neuronal (Kang et al., 2013), cambia la función cerebral en estado de reposo (Taylor et al., 2013), se activan áreas cerebrales que intervienen en la atención (Farb et al., 2013), la consciencia corporal (Siegel, 2007) y la regulación emocional (Tang y Posner, 2013). Todas estas capacidades se deterioran a lo largo de la EA, por tanto, entrenar mindfulness debería ser beneficioso para estos enfermos.

La EA es de etiología multifactorial y su curso se manifiesta mediante una sintomatología heterogénea con déficits cognitivos, funcionales y psicopatológicos. Diferentes estudios han mostrado que mindfulness es eficaz para reducir la psicopatología y mantener la funcionalidad (Lantz et al., 1997; McBee et al., 2004). Sin embargo, no se tienen suficientes datos consistentes de la utilidad de mindfulness sobre la cognición, que es el eje sobre el que se centra principalmente el tratamiento de la EA. Por tanto, para probar que mindfulness es útil debería modificar el curso del deterioro cognitivo. Este objetivo se ha analizado longitudinalmente, mediante una intervención de dos años, utilizando el "programa de estimulación para enfermos de Alzheimer basado en mindfulness (MBAS)". Para que MBAS sea eficaz debería mostrar un curso evolutivo diferencial al que produce la farmacología en el deterioro cognitivo. También se han investigado otros tratamientos no farmacológicos utilizados en la EA, estimulación cognitiva (EC) y relajación muscular progresiva (RMP) para conocer que aporta mindfulness en comparación a estos tratamientos. 


\section{MATERIAL Y MÉTODO}

\section{Sujetos}

Se realizó una evaluación neurológica integral (Alayón et al., 2009) de 502 personas mayores con diagnóstico de EA. La muestra inicial quedó constituida por 168 sujetos que cumplieron los criterios de inclusión. Los candidatos acudieron voluntariamente a la Unidad de detección de problemas de memoria de la Fundación Canaria Lidia García entre el año 2009 y 2011. En la figura 1 se puede observar la inclusión de los participantes en el estudio siguiendo las directrices de Moher et al. (2012) y Boutron et al. (2008).

Figura 1. Diagrama de flujo de las fases del ECA

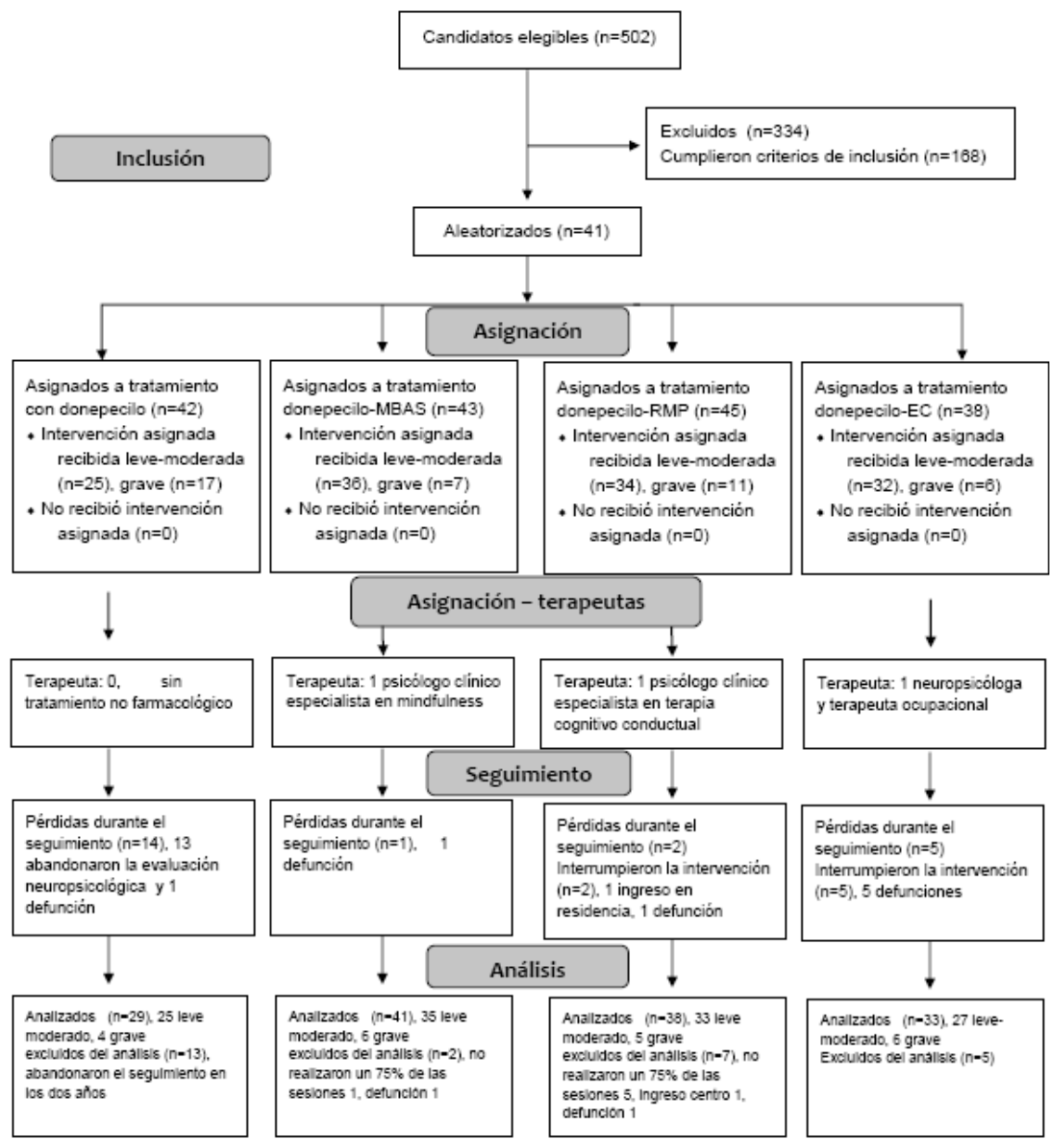




\section{Criterios de inclusión}

Personas mayores de 65 años con "EA Probable" según los criterios de la NINCDS-ARDA (McKhann et al., 1984) en fase leve o moderada con una puntuación en el MMSE mayor o igual a 18 puntos (Escribano-Aparicio et al., 1999), correspondiente a un estadio de deterioro global (GDS) de Reisberg et al. (1988) 3 o 4, o con un MMSE entre 15 y 17 puntos, correspondiente a un GDS 5. También tenían que vivir en su domicilio, contar con un familiar (pareja o hijas) que fuese el cuidador principal durante más de 5 horas diarias. Todos los sujetos estaban en tratamiento farmacológico con donepezilo.

\section{Instrumentos}

Se ha realizado una evaluación neuropsicológica con las escalas cognitivas incluidas en el CAMDEX-R (López-Pousa, 2003), el MMSE y el CAMCOG con sus diferentes subescalas: Orientación, Lenguaje, Memoria, Atención, Praxis, Cálculo, Pensamiento Abstracto y Percepción.

\section{Procedimiento}

Se desarrolló un ensayo clínico longitudinal controlado aleatorizado a doble ciego de dos años de duración, con medidas repetidas antes de iniciar el tratamiento y cuatro seguimientos semestrales. La evaluación era realizada por un neuropsicólogo que no intervenía en los tratamientos.

Se comparó la combinación de donepezilo y MBAS con otros tratamientos combinados utilizados tradicionalmente (EC y RMP) y un grupo control en tratamiento farmacológico. Los tres tratamientos experimentales fueron implementados por dos psicólogos clínicos y una neuropsicóloga independientes con más de diez años de experiencia.

El estudio ha sido aprobado por la comisión de ética de la Universidad de La Laguna y la participación en el mismo era voluntaria y requería la lectura, comprensión y firma de un consentimiento informado por parte del enfermo, el cuidador principal o su tutor legal. Cuando un enfermo cumplía los criterios de inclusión, la administrativa de la unidad de memoria se encargaba de asignarlos aleatoriamente a alguno de los grupos del estudio denominados A, B, C y D.

\section{Intervención}

Los tres grupos experimentales realizaron tres sesiones grupales de 90 minutos durante dos años, un total de 288 sesiones. El grupo experimental de EC realizó una intervención basada en el programa de psicoestimulación integral (Tárraga, 2010), el grupo de RMP desarrollo un programa basado en la propuesta de Amutio (1999), mientras que el MBAS se basó en un modelo combinado de prácticas derivadas del 
MBSR (Kabat-Zinn, 1990), el "Kirtan-Kriya" (Khalsa, 1998) y una adaptación de ejercicios de integración sensorial (Ayres, 1963) basados en mindfulness.

El entrenamiento de mindfulness se ha realizado mediante las prácticas formales desarrolladas en las sesiones grupales y con prácticas informales en la vida cotidiana entrenando los ejercicios de atención compartida con el cuidador principal.

Durante las sesiones de entrenamiento formal se ha preparado a los cuidadores y enfermos para la realización conjunta de ejercicios de atención focalizada y mindfulness. En las sesiones se repetía la siguiente metodología:

$1^{\circ}$. Orientación temporal y espacial: se comenzaba cada clase tomando consciencia del lugar en el que se está sentado y se verbalizaba el deseo de tener una sesión en armonía y sin dificultades, en la que se pudiese desarrollar una actitud de paz y bondad para practicar y estimular la atención al presente. Esta práctica es un ejercicio de "atención guiada Metta" (Kabat-Zinn, 1990).

$2^{\circ}$. Estiramientos de yoga en silla con ayudas de cintas de banda elástica.

$3^{\circ}$. Ejercicios de atención a la respiración y "escaneo corporal” siguiendo las recomendaciones de Kabat-Zinn (1990).

$4^{\circ}$. Entrenamiento con "Kirtan Kriya” (Khalsa, 1998).

$5^{\circ}$. Ejercicio guiado de atención focalizada basado en alguno de los cinco sentidos mediante visualizaciones, discriminación auditiva, aromaterapia, masajes (cara, manos, brazos), etc.

La segunda fase del programa es la realización de prácticas en casa mediante grabaciones de meditaciones guiadas en compañía del cuidador principal. También se proponía el desarrollo de prácticas informales compartidas con el cuidador para garantizar el desarrollo de las mismas. Se orientaba al enfermo y cuidador para que eligiesen 10 momentos durante el día donde pudieran practicar los ejercicios aprendidos. Se les recomendaba 3 minutos antes de las comidas, antes de levantarse y antes de dormirse y durante dos o tres paseos diarios en mañana o tarde.

\section{Análisis estadístico}

Se realizaron análisis estadísticos no paramétricos porque las variables dependientes del estudio no seguían una distribución normal. Primero se han analizado las diferencias generales intra-grupales con la prueba de Friedman, aplicando la prueba Wilcoxon con la corrección de Bonferroni $(p<0,005)$ cuando existían diferencias significativas para conocer partir de qué intervalo de seguimiento se producía un deterioro significativo. En segundo lugar, se ha valorado la existencia de diferencias existentes entre los grupos con las pruebas $\mathrm{H}$ de Kruskal-Wallis para analizar si existían diferencias generales, aplicando posteriormente la prueba Mann-Whitney con la corrección de Bonferoni $(p<0,008)$ para establecer entre que grupos existían diferencias. 
QUINTANA y QUINTANA. La eficacia de la estimulación para el Alzheimer basada en Mindfulness...

\section{RESULTADOS}

\section{Características sociodemográficas y clínicas de los participantes}

En la tabla 1 se presentan los datos descriptivos de los sujetos de los grupos experimentales y el grupo control previos al estudio. No se encontraron diferencias significativas en ninguna de las variables sociodemográficas. En las variables clínicas sólo se hallaron diferencias significativas en Dislipemias $(p=0,01)$ para los grupos con EA en fase leve-moderada.

Tabla 1. Características sociodemográficas y clínicas de la muestra previas al estudio

\begin{tabular}{|c|c|c|c|c|c|c|c|}
\hline \multicolumn{2}{|c|}{ Estadísticos de contraste Kruskal-Wallis } & \multicolumn{3}{|c|}{$\begin{array}{l}\text { Grupo de enfermedad de Alzheimer Leve } \\
\text { Moderada }\end{array}$} & \multicolumn{3}{|c|}{$\begin{array}{l}\text { Grupo de enfermedad de } \\
\text { Alzheimer Grave }\end{array}$} \\
\hline \multicolumn{2}{|c|}{ Variables sociodemográficas } & $N$ & $X^{2}$ & $\begin{array}{l}\text { Valor } \\
\text { de } p\end{array}$ & $N$ & $X^{2}$ & $\begin{array}{l}\text { Valor } \\
\text { de } p\end{array}$ \\
\hline \multirow{3}{*}{ Género } & & 120 & 1,74 & 0,62 & 22 & 2,064 & 0,559 \\
\hline & Mujeres & 66 & & & 15 & & \\
\hline & Hombres & 54 & & & 7 & & \\
\hline Edad & Media & $80,11 \pm 6,74$ & 9,24 & 0,25 & $84,11 \pm 5,08$ & 3,369 & 0,33 \\
\hline \multirow{4}{*}{ Estado Civil } & & & 1,72 & 0,63 & & 1,385 & 0,70 \\
\hline & Soltero & 5 & & & 2 & & \\
\hline & Casado & 73 & & & 13 & & \\
\hline & Viudo & 42 & & & 7 & & \\
\hline \multirow{3}{*}{ Población } & & & 1,89 & 0,60 & & 0,455 & 0,92 \\
\hline & Rural & 42 & & & 11 & & \\
\hline & Urbano & 78 & & & 11 & & \\
\hline \multirow{5}{*}{$\begin{array}{l}\text { Formación } \\
\text { académica }\end{array}$} & & & 4,27 & 0,23 & & 1,191 & 0,75 \\
\hline & Sin Formación & 1 & & & 1 & & \\
\hline & $\begin{array}{c}\text { Estudios } \\
\text { Primarios }\end{array}$ & 106 & & & 16 & & \\
\hline & Bachillerato & 21 & & & 4 & & \\
\hline & Universitarios & 12 & & & 1 & & \\
\hline \multirow{9}{*}{ Profesión } & & & 3,24 & 0,36 & & 2,672 & 0,44 \\
\hline & Ama de Casa & 75 & & & 13 & & \\
\hline & $\begin{array}{c}\text { Obrero no } \\
\text { especializado }\end{array}$ & 24 & & & 3 & & \\
\hline & $\begin{array}{c}\text { Obrero } \\
\text { especializado }\end{array}$ & 22 & & & 2 & & \\
\hline & Administrativo & 13 & & & 1 & & \\
\hline & Técnico medio & 7 & & & 1 & & \\
\hline & Maestro & 4 & & & 2 & & \\
\hline & $\begin{array}{c}\text { Otros } \\
\text { universitarios }\end{array}$ & 4 & & & & & \\
\hline & Empresario & 12 & & & & & \\
\hline Variables clínicas & $\%$ de enfermos & & $\mathrm{X}^{2}$ & $\begin{array}{l}\text { Valor } \\
\text { de p }\end{array}$ & $\begin{array}{c}\% \text { de } \\
\text { enfermos }\end{array}$ & $\mathrm{X}^{2}$ & $\begin{array}{l}\text { Valor } \\
\text { de p }\end{array}$ \\
\hline HTA & 51,6 & & 1,26 & 0,74 & 50 & 6,182 & 0,10 \\
\hline DM I & 3,1 & & 2,57 & 0,46 & 4,5 & 1,750 & 0,62 \\
\hline DMII & 11,2 & & 6,93 & 0,07 & 13,6 & 1,557 & 0,66 \\
\hline TSH & 8,1 & & 2,09 & 0,55 & 12 & 0,000 & 1,00 \\
\hline Dislipemias & 39,1 & & 9,94 & $0,01 *$ & 27,3 & 0,089 & 0,99 \\
\hline Psicopatología & 44,1 & & 3,19 & 0,36 & 40,9 & 4,006 & 0,26 \\
\hline $\begin{array}{l}\text { Consumo } \\
\text { antidepresivos }\end{array}$ & 31,7 & & 0,80 & 0,85 & 31,8 & 2,064 & 0,55 \\
\hline $\begin{array}{l}\text { Consumo } \\
\text { ansiolíticos }\end{array}$ & 31,7 & & 0,99 & 0,80 & 36,3 & 2,064 & 0,55 \\
\hline $\begin{array}{l}\text { Consumo } \\
\text { antipsicóticos }\end{array}$ & 3,1 & & 2,23 & 0,52 & 4,5 & 1,750 & 0,62 \\
\hline \multirow{6}{*}{ Biomarcador } & APO-E & & 3,62 & 0,30 & APO-E & 3,353 & 0,34 \\
\hline & $2 / 3$ & 2 & & & $3 / 3$ & 4 & \\
\hline & $3 / 3$ & 58 & & & $3 / 4$ & 9 & \\
\hline & $3 / 4$ & 24 & & & $4 / 3$ & 4 & \\
\hline & $4 / 3$ & 21 & & & $4 / 4$ & 5 & \\
\hline & $4 / 4$ & 15 & & & & & \\
\hline
\end{tabular}




\section{Deterioro Cognitivo leve moderado}

El análisis descriptivo de la puntuación global en las escalas cognitivas muestra como el MBAS presenta un deterioro menor a lo largo del estudio que el resto de los grupos como puede observarse en la tabla 2 con los porcentajes de deterioro global.

Los análisis intragrupo que muestran que MBAS presenta una estabilidad en las puntuaciones durante los 24 meses en el MMSE $(p=0,020)$ y en el CAMCOG $(p=0,387)$. Sin embargo el resto de grupos se deterioran significativamente. El grupo control después de los 12 meses de seguimiento en el MMSE $(p=0,000)$ y de los 6 meses en el CAMCOG $(p=0,001)$, EC a los 24 meses en el MMSE $(p=0,001)$ y a los 18 meses en el CAMCOG $(p=0,001)$. En último lugar, el grupo de RMP presenta una pérdida significativa de la puntuación después de los 12 meses en el MMSE $(p=0,001)$ y de los 24 meses en el CAMCOG $(p=0,000)$.

En la figura 2 y 3 se puede observar la evolución de la puntuación media en las escalas cognitivas a lo largo de todo el estudio. Estas figuras muestran una tendencia de mayor estabilidad en MBAS en comparación con el resto de los grupos que presentan una progresiva disminución de la puntuación.

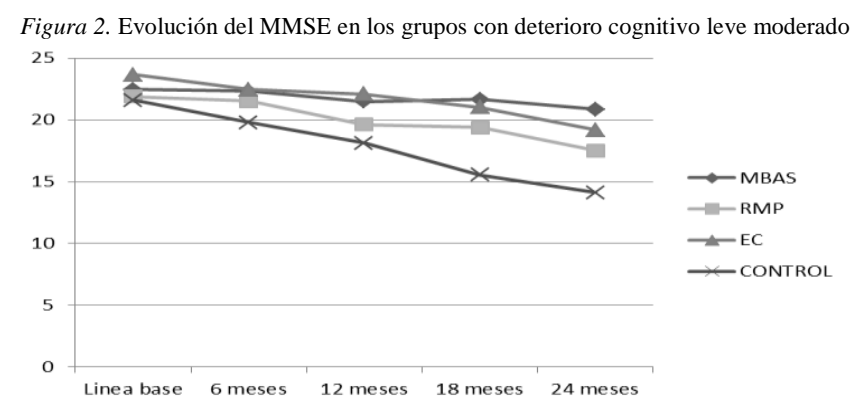

Figura 3. Evolución del CAMCOG en los grupos con deterioro cognitivo leve moderado

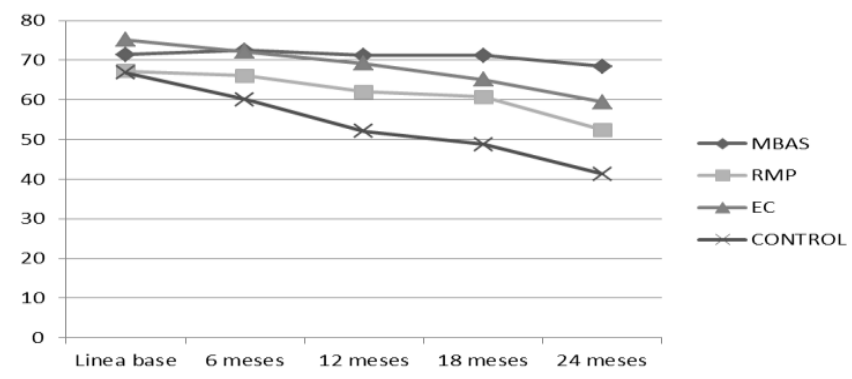

MBAS (Estimulación para el Alzheimer basado en mindfulness). RMP (Relajación muscular progresiva). EC (Estimulación Cognitiva) 
En la tabla 2 se presentan los estadísticos de contraste entre los grupos experimentales y el grupo control. Se puede observar como MBAS evoluciona significativamente mejor que EC y control. Sin embargo no presenta diferencias significativas con EC, salvo en las subescalas de abstracción y expresión del CAMCOG.

\section{Deterioro Cognitivo Grave}

Los resultados intragrupo mostraron como MBAS y EC han mantenido las puntuaciones estables a lo largo de los dos años de seguimiento con una $p>0,05$ en todas las medidas. Sin embargo, el grupo control (MMSE: $p=0,008$; CAMCOG: $p=0,001$ ) y el grupo de RMP (MMSE: $p=0,016$; CAMCOG: $p=0,007$ ) presentaron una pérdida significativa en la capacidad cognitiva.

El análisis entre los grupos experimentales y el control mostró que no se produjeron diferencias significativas en ninguna de las medidas. No obstante, la tendencia lineal muestra como MBAS presenta un incremento de la puntuación en el MMSE y CAMCOG hasta los 18 meses, mientras que la tendencia del resto de los grupos muestra un deterioro paulatino desde los 12 meses. Estos datos pueden verse en la figura 4 y 5 .

Figura 4. Evolución del MMSE en los grupos con deterioro cognitivo grave

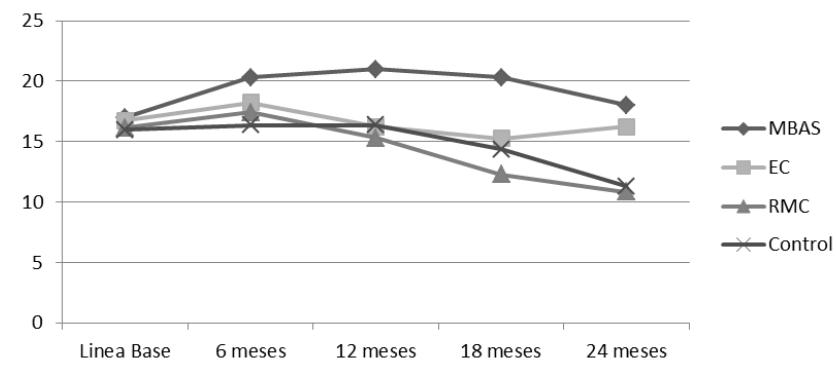

Figura 5. Evolución del CAMCOG en los grupos con deterioro cognitivo grave

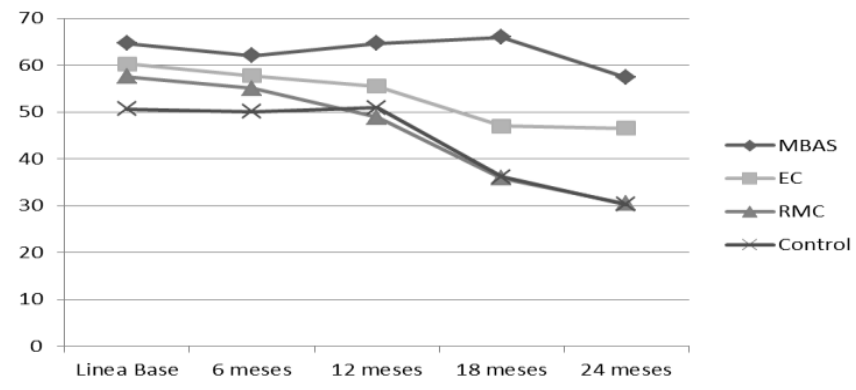

MBAS (Estimulación para el Alzheimer basado en mindfulness). RMP (Relajación muscular progresiva). EC (Estimulación Cognitiva) 


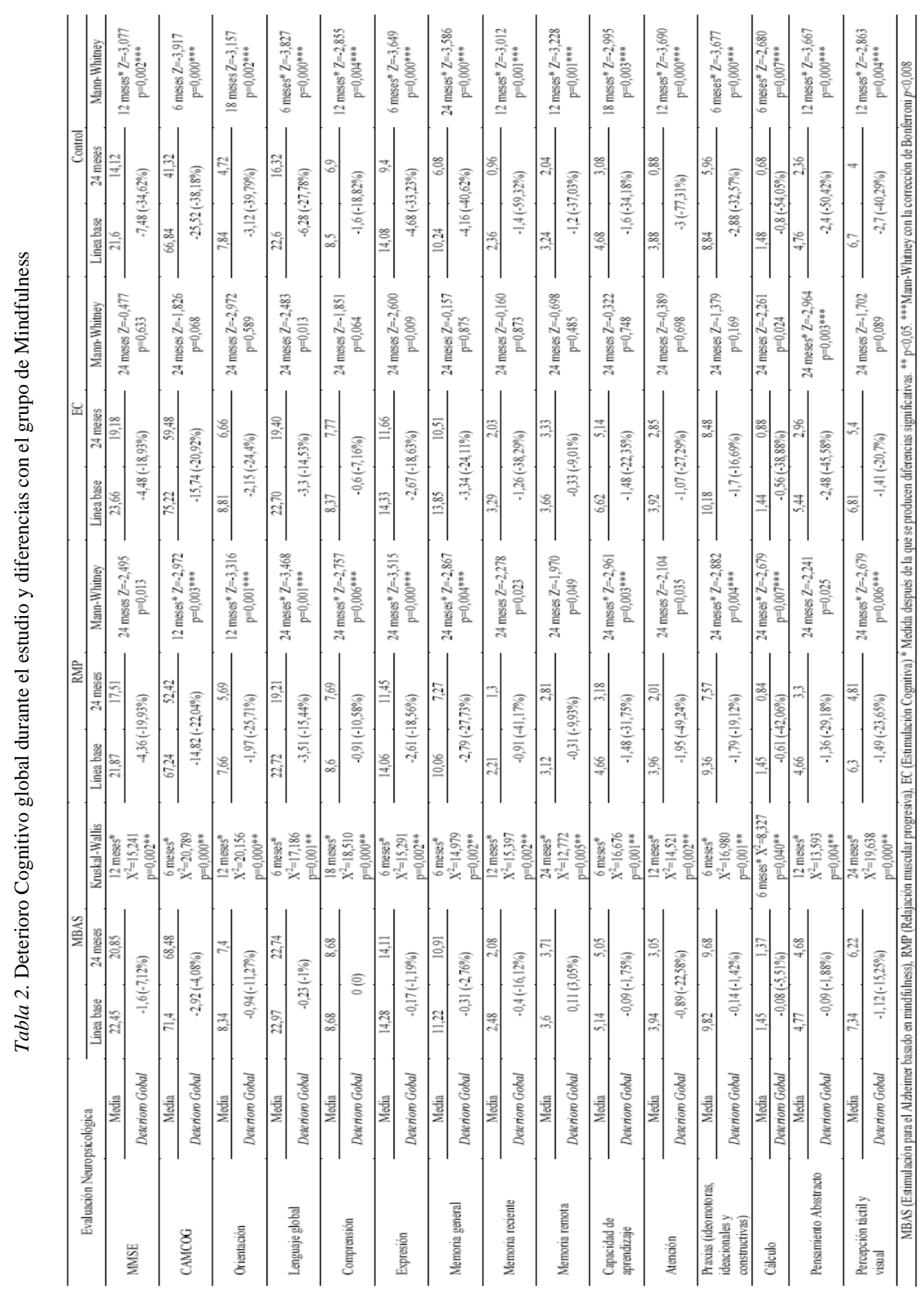




\section{DISCUSIÓN}

Este estudio longitudinal es un primer intento por demostrar que mindfulness es útil como tratamiento no farmacológico para frenar el deterioro cognitivo en la EA. Los resultados obtenidos muestran como la práctica de MBAS es efectiva en la fase leve en comparación con la utilización aislada de donepecilo desde los seis meses. También muestra una tendencia positiva en la fase avanzada, aunque no podemos concluir su efectividad debido a la falta de significación estadística por la limitación de sujetos en estadio grave.

La valoración global de los tratamientos no farmacológicos en fase levemoderada utilizados en este estudio muestran como el MBAS presenta una mejoría en el área cognitiva con el RMP desde los 12 meses y se muestra equivalente a un programa de EC clásico, mejorando sus puntuaciones de forma significativa en la capacidad de expresión y abstracción. Por tanto, estos datos parecen indicar que la práctica de MBAS permite obtener un efecto beneficioso en las áreas cognitivas de la EA inicial.

Un segundo aspecto a destacar, es que se produzcan diferencias significativas entre MBAS y EC en las subescalas del CAMCOG de expresión lingüística y capacidad de abstracción, pues la práctica continuada de mindfulness incide directamente en las capacidades del lóbulo frontal, favoreciendo ello probablemente la conservación de la expresión lingüística y la capacidad de pensamiento derivada del lenguaje. Este hallazgo es esperable con las prácticas de atención compartida entrenadas en MBAS, pues el desarrollo de los ejercicios de atención con el cuidador favorece la toma de decisiones del enfermo y, por tanto que este sea más autónomo. Enfatizamos estas diferencias con la estimulación cognitiva, pues a pesar de que pueden ser pequeñas y sólo al final del estudio, muestran como una práctica basada en mindfulness, en la que no se estimulan explícitamente las capacidades cognitivas, produce un beneficio global más significativo que el programa de estimulación cognitiva, que es el prototipo de intervención no farmacológica en la EA (Olazarán et al., 2010).

El último punto a destacar es el análisis de las puntuaciones intragrupos. En ellos se observa como el MBAS no presenta un deterioro significativo en el área cognitiva en dos años. Sin embargo, el resto de sujetos experimentales han mostrado un deterioro significativo de las capacidades cognitivas como en otros estudios que utilizan las mismas técnicas (Tárraga et al., 2010; Yanguas et al., 2006; Andersen et al, 2012). El dato más importante de estos resultados es que el MBAS ha mostrado una tasa de deterioro global diferente al resto de grupos, mostrando estabilidad en todas las puntuaciones. El resto de grupos experimentales y control han mostrado una tasa global de deterioro similar a la observada en otros estudios utilizando la misma escala (Conde et al., 2013). Estos datos periten considerar la posibilidad de que un tratamiento 
combinado entre donepezilo y mindfulness pueda cambiar el curso de la enfermedad, pues se frena el deterioro clínico de la misma.

En resumen, las conclusiones más importantes de este estudio son las siguientes: $1^{\circ}$ ) hasta donde nosotros sabemos este es el primer ECA longitudinal que aborda un efecto beneficioso de un programa basado en mindfulness sobre la función cognitiva de la enfermedad de Alzheimer. $2^{\circ}$ ) El MBAS combinado con donepezilo muestra un efecto terapéutico mayor sobre la cognición que otras combinaciones de tratamientos farmacológicos con relajación muscular o estimulación cognitiva o simplemente la prescripción farmacológica. $3^{\circ}$ ) Mindfulness puede ser integrado en los circuitos de atención a la EA como una nueva herramienta en los programas multimodales.

\section{REFERENCIAS}

Alayón, A., Quintana, D.J. y Morales, D. (2009). Implicación diagnóstica y terapéutica de la valoración neuropsicosocial integral en el paciente neurológico con demencia degenerativa. En J.L. Molinuevo y J.R. García (Coords.), Alzheimer 2009 transformando el presente en futuro. Madrid: Aula Médica.

Amutio, A. (1999). Teoría y práctica de la relajación: un nuevo sistema de entrenamiento. Barcelona: Martínez Roca.

Andersen F, Viitanen, M., Halvorsen, D.S., Straume, B., Wilsgaard, T. y Engstad, T.A. (2012).The effect of stimulation therapy and donepezil on cognitive function in Alzheimer's disease. A community based RCT with a two-by-two factorial design. BMC Neurol, 19, 12-59.

Ayres, A.J. (1963). The development of perceptual-motor abilities: A theoretical basis for treatment of dysfunction. Am J Occup Ther, 17, 221-225.

Bishop, S.R., Shapiro, L.M., Carlson, S., Anderson, L., Carmody, N., Segal, et al. (2004). Mindfulness: A Proposed Operational Definition. Clinical Psychology: Science and Practice, 11, 230-241.

Boutron, I., Moher, D., Altman, D.G., Schulz, K., Ravaud, P., for the CONSORT Group (2008). Methods and Processes of the CONSORT Group: Example of an Extension for Trials Assessing Nonpharmacologic Treatments. Ann Intern Med, W60-W67.

Conde-Sala, J.L., Garre-Olmo, J., Vilalta-Franch, J., Llinàs-Reglà, J., Turro-Garriga, O., LozanoGallego, M. y López-Pousa, S. (2013). Cognitive decline in Alzheimer's disease. A follow three or more years of a sample of patients. Revista de Neurología, 56(12), 593600.

Escribano-Aparicio, M.V., Pérez-Dively, M., García-García, F.J., Pérez-Martín, A., Romero, L., Ferrer, et al. (1999). Validación del MMSE de Folstein en una población española de bajo nivel educativo. Rev Esp Geriatr Gerontol, 34,319-26.

Hölzel, B.K., Carmody, J., Vangel, M., Congleton, C., Yerramsetti, S.M., Gard, T. y Lazar, S.W. (2011). Mindfulness practice leads to increases in regional brain gray matter density. Psychiatry Research, 191(1), 36-43.

Kabat-Zinn, J. (1990). Full catastrophe living. Using the Wisdom of Your Body and Mind to Face Stress, Pain, and Illness. Delta Book Publishing. New York.

Kang, D.H., Jo, H.J., Jung, W.H., Kim, S.H., Jung, Y.H., Choi, C.H. y Kwon, J.S. (2013). The effect of meditation on brain structure: cortical thickness mapping and diffusion tensor imaging. Social Cognitive and Affective Neuroscience, 8(1), 27-33. 
Khalsa, D.S. (1998). Integrated medicine and the prevention and reversal of memory loss. Alternative Therapies in Health and Medicine, 4(6), 38-43.

Lantz, M.S., Buchalter, E.N. y McBee, L. (1997). The Wellness Group: a novel intervention for coping with disruptive behavior among [corrected] elderly nursing home residents. The Gerontologist, 37(4), 551-556.

Lazar, S., Kerr, C.E., Wasserman, R.H., Gray, J.R., Greve, D.N., Treadway, et al. (2005). Meditation experience is associated with increased cortical thickness. Neuroreport, 16(17), 1893-1897.

López-Pousa, S. (2003). Adaptación Española de la Prueba de exploración Cambridge revisada para la valoración de los trastornos mentales en la vejez de Roth et al. Madrid: TEA.

Marciniak, R., Sheardova, K., Čermáková, P., Hudeček, D., Šumec, R. y Hort (2014). Effect of meditation on cognitive functions in context of aging and neurodegenerative diseases. Front. Behav. Neuroscience, 27.

McBee, L., Westreich, L. y Likourezos, A. (2004). A psychoeducational relaxation group for pain and stress in the nursing home. Journal of Social Work in Long Term Care, 3, 15-28.

McKhann, G., Drachman, D., Folstein, M., Katzman, R., Price, D. y Stadlan, E.M. (1984). Clinical diagnosis of Alzheimer's disease: report of the NINCDS-ADRDA Work Group under the auspices of Department of Health and Human Services Task Force on Alzheimer's disease. Neurology, 34(7), 939-44.

Moher, D., Hopewell, S., Schulz, K.F., Montori, V., Gøtzsche, P.C. y Devereaux, P.J. (2012). Consort 2010 explanation and elaboration: updated guidelines for reporting parallel group randomised trials. International Journal of Surgery, 10(1), 28-55.

Olazarán, J., Reisberg, B., Clare, L., Cruz, I., Peña-Casanova, J., Del Ser, T. y Muñiz, R. (2010). Non pharmacological therapies in Alzheimer's disease: a systematic review of efficacy. Dementia and Geriatric Cognitive Disorders, 30(2), 161-178

Reisberg, B., Ferris, S.H., de Leon, M.J. y Crook, T. (1988). Global Deterioration Scale (GDS). Psychopharmacol Bull, 24(4), 661-3.

Siegel, D.J. (2007). The mindful brain: reflection and attunement in the cultivation of well-being. New York: W.W. Norton.

Tárraga, J.L. (2010). Tratamiento no farmacológico en las demencias. En R. Alberca y S. LópezPousa (Eds.), Enfermedad de Alzheimer y otras demencias (pp. 83-95). Madrid: Panamericana.

Tang, Y. y Posner, M.I. (2013). Special issue on mindfulness neuroscience. Social Cognitive and Affective Neuroscience, 8(1), 1-3.

Taylor, V.A., Daneault, V., Grant, J., Scavone, G., Breton, E., Roffe-Vidal, S. y Beauregard, M. (2013). Impact of meditation training on the default mode network during a restful state. Social Cognitive and Affective Neuroscience, 8(1), 4-14.

Wells, R.E., Yeh, G.Y., Kerr, C., Wolkin, J., Davis, R.B., Tan, Y. y Kong, J. (2013). Meditation's impact on default mode network \& hippocampus in mild cognitive impairment: a pilot study. Neuroscience letters.

Yanguas, J.J., Buiza, C., Etxeberría, I., Galdona, N., González M.F., Arriola, E., López, A. y Urdaneta, E. (2006). Estudio Longitudinal Donostia de enfermedad de Alzheimer. Madrid: Tempora.

Recibido: 15 de abril de 2014 Recepción Modificaciones: 26 de mayo de 2014 Aceptado: 28 de mayo de 2014 